\title{
Tratamento da Distonia Laríngea com Toxina Botulínica
}

\section{Treatment of Laryngeal Dystonia with Botulinum Toxin}

Pedro Valente ${ }^{1}$, Isabel Gomes Pintoํ․, Francisco Monteiro ${ }^{1}$, Eugénia Castro ${ }^{2,3}$, Artur Condé 4

\section{RESUMO}

INTRODUÇÃO: A distonia laríngea focal engloba um conjunto de processos patológicos, caracterizados por contrações involuntárias e intermitentes dos músculos intrínsecos da laringe, que podem prejudicar predominantemente a fonação ou a respiração. A injeção de toxina botulínica nos músculos afetados provoca uma paralisia transitória, permitindo um bom controlo sintomático nestes pacientes.

MÉTODOS: Análise retrospetiva dos doentes com distonia laríngea submetidos a tratamento com toxina botulínica no Serviço de ORL do CHVNG/E, entre janeiro/2011 e dezembro/2016.

RESULTADOS: Durante o período de 6 anos foram identificados 11 doentes com distonia laríngea ( $91 \%$ sexo feminino, idade média 42 anos). Neste grupo 45,5\% apresentavam distonia laríngea fonatória de tipo adutor e 9,1\% de tipo misto, enquanto 45,5\% apresentavam distonia laríngea respiratória.

Estes pacientes foram submetidos a 43 injeções de toxina botulínica (média de quatro procedimentos/doente), em que 39,5\% foram realizadas em ambulatório sob controlo eletromiográfico e 60,5\% ocorreram sob anestesia geral. As injeções foram unilaterais em 30,2\% e bilaterais em 67,4\%. A dose média injetada foi de 11,7 U por sessão. A meIhoria sintomática parcial ou total ocorreu em 83,7\%, com ocorrência de episódios de engasgamento para líquidos após $20,9 \%$ dos procedimentos. A mediana de duração do benefício da toxina botulínica foi de 7 meses.

DISCUSSÃO E CONCLUSÃO: A distonia laríngea tem origem em alterações neurológicas crónicas do processamento motor central e, devido às dificuldades no seu diagnóstico, ocorrem consideráveis atrasos no início de uma intervenção terapêutica apropriada. Entre as inúmeras propostas de tratamentos médicos e cirúrgicos, a toxina botulínica mantém-se, na atualidade, como o tratamento de primeira linha, embora a otimização individual da dose terapêutica possa condicionar os resultados iniciais.

PALAVRAS-CHAVE: Distonia/tratamento; Doenças da Laringe/tratamento; Toxina Botulínica/uso terapêutico

1. Interno de Formação Específica do Serviço de Otorrinolaringologia do Centro Hospitalar de Vila Nova de Gaia/Espinho, Porto, Portugal.

2. Assistente Hospitalar Graduada do Serviço de Otorrinolaringologia do Centro Hospitalar de Vila Nova de Gaia/Espinho, Porto, Portugal.

3. Coordenadora da Unidade da Voz do Hospital CUF Porto, Porto, Portugal.

4. Diretor do Serviço de Otorrinolaringologia do Centro Hospitalar de Vila Nova de Gaia/Espinho, Porto, Portugal.

Recebido: 04/02/2019 - Aceite: 13/03/2019 


\section{ABSTRACT}

INTRODUCTION: Focal laryngeal dystonia encompasses a set of pathological processes, characterized by involuntary and intermittent contractions of the internal laryngeal muscles, which can affect predominantly phonatory or respiratory tasks. The injection of botulinum toxin into the affected muscles causes a transient paralysis, allowing a good symptomatic control in these patients.

METHODS: Retrospective analysis of patients with laryngeal dystonia undergoing treatment with botulinum toxin in the Otolaryngology department of our institution between January/2011 and December/2016.

RESULTS: During the 6-year interval, 11 patients with laryngeal dystonia were identified (91\% female, mean age 42 years). In this group, 45.5\% had adductor type laryngeal dystonia and 9.1\% mixed type, while $45.5 \%$ presented respiratory laryngeal dystonia.

These patients underwent 43 injections of botulinum toxin (mean of four procedures per patient), in which 39.5\% were performed in an outpatient clinic under electromyographic control and $60.5 \%$ under general anesthesia. The injections were unilateral in 30.2\% and bilateral in 67.4\%. The mean dose injected was $11.7 \mathrm{U}$ per session. Partial or total symptomatic improvement occurred in $83.7 \%$, with temporary dysphagia after $20.9 \%$ of the procedures. The median duration of botulinum toxin effect was 7 months.

DISCUSSION AND CONCLUSION: Laryngeal dystonia is thought to arise from chronic neurological disorders of central motor processing and considerable delays still occur before appropriate therapeutic intervention, due to difficulties in achieving the correct diagnosis. Among several medical and surgical treatments, botulinum toxin remains nowadays as the first-line treatment, although individual optimization of the therapeutic dose may limit the initial results.

KEYWORDS: Botulinum Toxins/therapeutic use; Dystonia/drug therapy; Laryngeal Diseases/drug therapy

\section{INTRODUÇÃO}

A distonia laríngea ( $D L$ ), na qual se inclui a disfonia espasmódica, é uma patologia crónica caracterizada por contrações involuntárias e inapropriadas dos músculos intrínsecos da laringe, induzidas por tarefas específicas.

A evolução do conhecimento neurobiológico conduziu a múltiplas alterações da nomenclatura desta doença, inicialmente percecionada como uma alteração psicogénica por múltiplos autores durante o século XIX e início do século XX.1,2 Considerando o insucesso da psicoterapia no tratamento sintomático dos indivíduos afetados, em conjunto com revisões de entrevistas psiquiátricas ${ }^{3}$ e estudos de eletromiografia laríngea, ${ }^{4}$ a DL foi reclassificada como uma distonia focal na década de 1980 por diversos grupos de investigação. ${ }^{4-6}$

Em termos epidemiológicos, a DL é uma patologia rara, considerada a quarta forma mais prevalente de distonia focal, com prevalência estimada de 6,7 casos por miIhão. ${ }^{7}$ A vasta maioria dos indivíduos afetados é do sexo feminino, englobando até $80 \%$ dos casos em algumas séries. ${ }^{8}$

Os mecanismos patofisiológicos da DL permanecem desconhecidos, embora um conjunto de alterações no processamento motor central tenha sido demonstrado recentemente nestes pacientes, afetando diversas regiões cerebrais como o córtex cerebral, cerebelo, tálamo e tronco cerebral. ${ }^{9}$ No que diz respeito à etiologia, a maioria dos casos são esporádicos e, apesar de cerca de 10\% dos indivíduos reportarem uma história familiar de distonia, ${ }^{10}$ os genes responsáveis pela $\mathrm{DL}$ ainda não foram identificados.

Clinicamente, a DL pode manifestar-se durante o esforço fonatório, particularmente durante os movimentos de hiperadução (encerramento) ou hiperabdução (abertura) das cordas vocais. A maioria dos casos de DL são de tipo adutor, no qual ocorrem contrações involuntárias e intermitentes dos músculos laríngeos adutores que originam uma voz tensa e estrangulada com quebras vocais frequentes. No caso da DL de tipo abdutor, a hiperreatividade dos músculos abdutores conduz a uma voz soprosa, com quebras na fluência vocal. A DL de tipo misto engloba os raros casos que apresentam características de ambos os tipos. A DL respiratória é determinada pela ocorrência de espasmos dos músculos adutores durante a inspiração, ${ }^{11}$ que pode conduzir a estridor e pausas respiratórias inadequadas devido a movimentos inspiratórios paradoxais das cordas vocais. Esta forma de DL não envolve a fonação e geralmente desaparece com a voz cantada, riso e durante o sono, ${ }^{12}$ como se verifica também nas restantes variantes de distonia laríngea. 
O diagnóstico da DL deve assentar numa história médica detalhada, avaliação das características fonatórias e estudo videolaringoestroboscópico por um otorrinolaringologista, em conjunto com um exame neurológico, para deteção de eventuais alterações distónicas em outros segmentos corporais (distonia segmentar ou generalizada) e exclusão de doenças neurológicas associadas. ${ }^{13} \mathrm{~A}$ eletromiografia laríngea (EMGL) não é considerada um exame definitivo para o diagnóstico, embora alguns autores refiram a existência de padrões sugestivos como o aumento nos períodos de latência, amplitude e frequência dos potenciais de ativação muscular. ${ }^{14}$

Após experiências pouco eficazes com terapias farmacológicas ou alternativas cirúrgicas, foi realizado o primeiro ensaio com aplicação de toxina botulínica (TB) em três doentes com DL em 1984. ${ }^{15}$ Deste então, múltiplos autores têm estudado a eficácia da TB no tratamento da DL, com evidência fortemente positiva em favor desta terapia, que é atualmente considerada como tratamento de eleição na $\mathrm{DL}^{16}$ e recomendada pela Academia Americana de Otorrinolaringologia. ${ }^{17}$

A TB é produzida pela bactéria Clostridium botulinum e exerce o seu efeito através da inativação de proteínas de fusão, responsáveis pela libertação de acetilcolina na fenda sináptica, impedindo a despolarização do terminal pós-sináptico, o que resulta em fraqueza muscular. Foram identificados sete serotipos de TB diferentes, sendo o tipo A o mais potente e mais utilizado clinicamente. Quando aplicada nos músculos laríngeos, a TB diminui a atividade muscular; no entanto, a sua ação local parece não explicar totalmente a sua eficácia clínica, o que sugere a existência de um conjunto de mecanismos de ação não-clássicos que modulam diretamente o sistema nervoso central. ${ }^{18-20}$ A aplicação de TB pode ser realizada através de microlaringoscopia direta em suspensão ou por punção percutânea, monitorizada por EMGL ou com visualização direta por nasofibrolaringoscopia. Os efeitos clínicos têm uma duração média entre 3-6 meses (que depende da dose injetada), e a reinjeção é habitualmente necessária para manutenção dos benefícios sintomáticos. ${ }^{10}$

O objetivo deste artigo é relatar a experiência clínica de uma série de pacientes com DL, observados em consulta especializada de patologia vocal no Centro Hospitalar de Vila Nova de Gaia/Espinho (CHVNG/E), durante um período de seis anos, e descrever as suas características clínicas e resultados obtidos com o tratamento com TB.

\section{MÉTODOS}

Analisaram-se retrospetivamente os processos clínicos eletrónicos de todos os doentes com DL avaliados em Consulta de Voz e submetidos a tratamento com TB no Serviço de Otorrinolaringologia do CHVNG/E, entre janeiro de 2011 e dezembro de 2016.

O diagnóstico de DL baseou-se na avaliação clínica realizada por um otorrinolaringologista e neurologista, confirmado por videolaringoestroboscopia com identificação dos grupos musculares afetados. A EMGL realizou-se em todos os pacientes, permitindo avaliar e excluir outras neuropatias e identificar áreas com maior número de unidades motoras, guiando a injeção de TB.

As injeções de TB (Botox ${ }^{\circledR}$; Allergan, Irvine, CA, EUA) foram realizadas pelo mesmo cirurgião (EC), sob anestesia geral, com visualização direta da laringe através de microlaringoscopia em suspensão ou, em ambulatório, utilizando uma via percutânea (transcricotiroideia) com controlo eletromiográfico. Nesta última, é utilizada uma agulha de calibre 27G, revestida por Teflon ${ }^{\circledR}$, para puncionar a membrana cricrotiroideia e avançar com angulação superior e lateral em direção ao complexo muscular tiroaritenoideu/cricoaritenoideu lateral. A posição da agulha é depois validada pedindo ao paciente para emitir o som da vogal /e/, que provoca um aumento brusco e sustentado de atividade eletromiográfica. A dose e realização de injeção uni ou bilateralmente foi determinada por decisão clínica e preferência do paciente, de acordo com as respostas obtidas previamente.

Os dados recolhidos incluíram as características do doente e da patologia, dados relativos à injeção de TB (dose média, lateralidade, músculos abrangidos), índice GRBAS ${ }^{21}$ prévio à primeira injeção e após otimização da dose individual, qualidade vocal pós-injeção (ausência melhoria, melhoria parcial, melhoria marcada/quase normal), duração média de ação da TB e efeitos laterais.

\section{RESULTADOS}

Identificaram-se um total de onze doentes com o diagnóstico de DL, durante o período de seis anos, dez do sexo feminino e um do sexo masculino, com idades compreendidas entre os 20 e os 77 anos e uma idade média de 41,9 anos (d.p. 19,5 anos). Quanto aos tipos de DL identificados, 45,5\% ( $n=5)$ apresentavam DL fonatória de tipo adutor e $9,1 \%(n=1)$ de tipo misto, enquanto $45,5 \%$ ( $n=5)$ apresentavam DL respiratória. Três doentes apresentavam associadamente distonia generalizada, cervical ou síndrome de Meige (caracterizada pela ocorrência conjunta de blefarospasmo e distonia 
oromandibular). Nenhum doente apresentava história familiar de DL.

A queixa inicial destes pacientes foi disfonia em seis casos (54,5\%) e dispneia em cinco casos (45,5\%). Ao exame objetivo, evidenciaram-se alterações na laringoscopia em quatro doentes com visualização de movimento paradoxal das cordas vocais e, na videoestroboscopia, verificou-se a existência de constrição glótica ou supraglótica em seis casos. A realização da EMGL permitiu identificar em alguns casos alterações sugestivas de um padrão distónico.

Estes pacientes foram submetidos a um conjunto de 43 injeções de TB (média de quatro procedimentos por caso), que foram realizadas em ambulatório sob controlo eletromiográfico em 39,5\% ( $n=17)$ dos casos e em $60,5 \%$ ( $n=26$ ) sob anestesia geral. As injeções foram unilaterais em 31,0\% ( $n=13$ ) e bilaterais em 69,0\% ( $n=29$ ) dos procedimentos. Os músculos mais frequentemente abrangidos foram o tiroaritenoideu (46,5\%; $\mathrm{n}=20$ ) e o complexo formado pelo tiroaritenoideu, cricoaritenoideu lateral e banda ventricular (23,3\%; $\mathrm{n}=10$ ). A dose média injetada foi de 11,7 U por sessão (d.p. 6,3 U). Na Tabela 1 podem ser consultados os dados globais relativos ao conjunto de procedimentos efetuados por paciente, assim como as respetivas informações clínicas.

Em termos de qualidade vocal pós-operatória reportada pelo paciente, a melhoria sintomática parcial ou total ocorreu após 83,7\% ( $n=36)$ procedimentos, sendo que, em 9,3\% das injeções o paciente considerou ter ocorrido ausência de benefício com a dose injetada. Em termos de efeitos laterais da TB, foram registados fenómenos de engasgamento para líquidos após 20,9\% dos procedimentos ( $n=9$ ). A maioria dos doentes relatou um período transitório de aumento de soprosidade na voz, com duração máxima de duas semanas, após a intervenção. Não foram registadas complicações derivadas da infiltração laríngea de TB, ou desenvolvimento de tolerância à TB. A mediana de duração de efeito da TB foi de sete meses (intervalo interquartil: 5-11 meses).

\section{DISCUSSÃO}

A DL é uma patologia pouco frequente, caracterizada por movimentos involuntários anormais das cordas vocais, com início na idade adulta e classificada como uma distonia focal.

Nesta série, tal como descrito na literatura, a maior parte dos doentes eram do sexo feminino e adultos. De referir a existência de dois casos de DL associada a distonia segmentar (cervical e síndrome de Meige) e um caso de distonia generalizada.

No que diz respeito aos tipos de distonia identificados, a DL de tipo adutor e respiratório formaram a maioria dos casos. O número aparentemente elevado de casos de DL respiratória é justificável pelo facto deste tipo específico, caracterizado por sintomas respiratórios mais evidentes, motivar a procura de cuidados médicos pelos indivíduos que, em alguns casos, são referenciados de outros centros para a Consulta de Voz do CHVNG/E.

TABELA 1. Dados clínicos dos pacientes com DL e características globais dos tratamentos efetuados.

\begin{tabular}{|c|c|c|c|c|c|c|c|c|c|}
\hline Paciente & $\begin{array}{l}\text { Idade } \\
\text { (anos) }\end{array}$ & Sexo & $\begin{array}{l}\text { Padrão } \\
\text { Distonia }\end{array}$ & $\begin{array}{c}\text { Tipo } \\
\text { Distonia } \\
\text { Laríngea }\end{array}$ & $\begin{array}{l}\text { Dose Média } \\
\text { TB } \\
\text { Injetada (U) }\end{array}$ & $\begin{array}{l}\text { Lateralidade } \\
\text { Injeção TB }\end{array}$ & $\begin{array}{l}\text { Músculos } \\
\text { Injetados }\end{array}$ & $\begin{array}{l}\text { GRBAS total } \\
\text { pré-TB* }\end{array}$ & $\begin{array}{l}\text { GRBAS total } \\
\text { pós-TB }^{\dagger}\end{array}$ \\
\hline 1 & 77 & $\mathrm{~F}$ & Fo & Resp & 12,25 & Uni/bilateral & $\mathrm{TA}+\mathrm{CAL}$ & 4 & 2 \\
\hline 2 & 20 & M & Fo & Resp & 10,58 & Bilateral & $\mathrm{TA}+\mathrm{CAL}+\mathrm{BV}$ & 9 & 6 \\
\hline 3 & 38 & F & Fo & $\mathrm{Ad}$ & 12,12 & Bilateral & TA & 6 & 2 \\
\hline 4 & 24 & $\mathrm{~F}$ & Fo & Resp & 6,66 & Bilateral & TA & 5 & 3 \\
\hline 5 & 56 & F & Fo & Ad & 7,75 & Uni/bilateral & TA & 9 & 2 \\
\hline 6 & 21 & F & Fo & $\mathrm{Ad}$ & 6,25 & Bilateral & TA & 6 & 2 \\
\hline 7 & 62 & F & Seg & $\mathrm{Ad}$ & 8,91 & Bilateral & $\mathrm{TA}+\mathrm{CF}$ & 9 & 3 \\
\hline 8 & 25 & F & Fo & Resp & 10,25 & Bilateral & $\mathrm{TA}+\mathrm{CAL}+\mathrm{BV}$ & 10 & 7 \\
\hline 9 & 61 & F & Ge & $\mathrm{Ad}$ & 3,25 & Unilateral & TA & 4 & 1 \\
\hline 10 & 42 & F & Fo & Resp & 22,50 & Bilateral & TA & 8 & 5 \\
\hline 11 & 35 & $\mathrm{~F}$ & Seg & Mi & 17,56 & Bilateral & $\begin{array}{c}\mathrm{TA}+\mathrm{CAL}+\mathrm{B}- \\
V+\mathrm{CF}\end{array}$ & 9 & 3 \\
\hline
\end{tabular}

F: Feminino; M: Masculino; Fo: Focal; Ge: Generalizada; Seg: Segmentar; Resp: Respiratória; Ad: Adutora; Mi: Mista; TB: Toxina botulínica; TA: Tiroaritenoideu; CAL: Cricoaritenoideu lateral; BV: Banda ventricular; CF: Músculos constritores da faringe.

* GRBAS total prévio à primeira injeção de TB; † GRBAS total após otimização individual da dose de TB. 
O diagnóstico adequado de DL é complexo, sendo facilmente confundida com outras entidades como a disfonia por tensão muscular, condicionando atraso de vários anos no diagnóstico. ${ }^{22}$ A avaliação especializada por um grupo multidisciplinar, associada ao exame videolaringoestroboscópico e realização de EMGL, permitiu definir o diagnóstico neste conjunto de doentes.

Em termos de tratamento, vários procedimentos cirúrgicos têm sido propostos na DL, nomeadamente a desnervação-reinervação seletiva dos músculos adutores laríngeos, idealmente realizada em doentes com um diagnóstico preciso de DL de tipo adutor que não apresentam benefício ou não toleram o tratamento com TB, ${ }^{23}$ que apresenta taxas de insucesso entre 10\% e 12\%. ${ }^{24} \mathrm{~A}$ realização de miomectomia tiroaritenoideia, com abordagem transcervical ${ }^{25}$ ou por via transoral associada a neurectomia com laser $\mathrm{CO}_{2},{ }^{26}$ é outra potencial opção de longa-duração na DL de tipo adutor com resultados cirúrgicos encorajadores, apesar da existência de um número significativo de doentes que necessitaram de cirurgia de revisão. ${ }^{27}$ Por último, a opção da tiroplastia tipo II reportada inicialmente por Isshiki et al ${ }^{28}$ tem sido identificada como um tratamento potencialmente eficaz a longo-prazo.

Atualmente, o tratamento de eleição na DL envolve a aplicação intramuscular de TB nos grupos musculares afetados e apresenta taxas de sucesso de 90\% na DL adutora; contudo a sua eficácia na DL abdutora e respiratória é inferior, aproximadamente 70\% e 55\% respetivamente. $^{29}$

Dada a ausência de protocolos internacionais, a dose utilizada na terapia com TB e a aplicação de forma bilateral ou unilateral varia caso a caso, conforme a experiência de cada instituição e o perfil do doente. Ambas as técnicas de injeção, uni e bilateral, apresentaram bons resultados, com vantagem para a aplicação bilateral em termos de qualidade vocal e duração de efeito. ${ }^{30}$ No entanto, existe controvérsia em relação à dose e lateralidade, tendo sido descrita a utilização de doses unilaterais com resultados consistentes em termos sintomáticos associada a um perfil de menores efeitos adversos. ${ }^{31,32}$ Na nossa instituição tem sido dada preferência à realização de injeções bilaterais, com uma dose mais elevada (2,5-5,0 U) unilateralmente e uma dose inferior (1,5 U) na corda vocal contralateral, favorecendo o efeito bilateral e maior longevidade do efeito terapêutico, reduzindo o número médio de injeções necessárias por doente.

A evidência de hiperadução supraglótica compensadora em até 25\% dos pacientes com DL de tipo adutor, que pode condicionar um efeito subótimo da TB, levou à apli- cação de TB nos grupos musculares supraglóticos, com benefício significativo nestes pacientes. ${ }^{33}$ Na nossa série foram distribuídas doses de TB nas bandas ventriculares em dez procedimentos com o objetivo de reduzir a hiperadução supraglótica e aliviar o período de maior soprosidade após a aplicação de TB. ${ }^{34}$

A perceção subjetiva do doente sobre a função vocal é um indicador crítico do resultado do tratamento. Neste estudo, a avaliação do sucesso da aplicação de TB baseou-se na perceção do paciente sobre a sua qualidade vocal pós-procedimento. Embora fosse desejável a quantificação objetiva das alterações vocais, a natureza retrospetiva deste estudo impediu a recolha desses parâmetros. Assim, foi reportada uma melhoria na qualidade vocal após 83,7\% procedimentos, que considerando a elevada proporção de casos de DL respiratória, é um resultado que está de acordo com as séries existentes na literatura. 12,29 A otimização da dose individual pode requerer, em média, três procedimentos, o que permite explicar que os restantes casos com ausência inicial de melhoria na qualidade vocal tenham obtido benefício em procedimentos subsequentes, após ajuste de dose.

Em termos de efeitos laterais, as injeções de TB estão associadas com o aumento da soprosidade na voz e disfagia devido a insuficiência glótica condicionada pela paresia temporária. ${ }^{29} \mathrm{Na}$ experiência dos autores, a disfagia foi mais intensa nos pacientes submetidos a injeção supraglótica e ao nível dos constritores faríngeos. $\bigcirc$ ajuste da dose, distribuição e intervalos entre as injeções pode diminuir a frequência destes eventos adversos. Outros efeitos possíveis incluem hemorragia, edema das cordas vocais e sensações de irritação laríngea. $\bigcirc$ desenvolvimento de resistência secundária ao efeito da TB pode ocorrer raramente. ${ }^{29}$ Nesta série foram reportados fenómenos transitórios de aumento de soprosidade e de engasgamento para líquidos após 20,9\% dos procedimentos, compatível com os valores apresentados na literatura. ${ }^{10}$

\section{CONCLUSÃO}

Apesar de não constituir uma condição fatal, a distonia laríngea condiciona alterações significativas na qualidade e fluência vocal e acarreta perda de qualidade de vida. ${ }^{35}$ A aplicação de toxina botulínica é o tratamento recomendado internacionalmente e permite bons resultados vocais. A compreensão do perfil de efeitos laterais associados à aplicação de toxina botulínica permite o ajuste individualizado da terapêutica, que deve ser englobado num processo de decisão partilhado pelo médico e doente. 
CONFLITOS DE INTERESSE: Os autores declaram não ter qualquer conflito de interesse na realização do presente trabalho.

FONTES DE FINANCIAMENTO: Não houve qualquer fonte de financiamento na realização do presente trabalho.

CONFIDENCIALIDADE DOS DADOS: Os autores declaram ter seguido os protocolos da sua instituição acerca da publicação dos dados de doentes.

PROTEÇÃO DE PESSOAS E ANIMAIS: Os autores declaram que os procedimentos seguidos na elaboração do presente trabalho estão em conformidade com as normas das comissões de investigação clínica e de ética, bem como da declaração de Helsínquia e da Associação Médica Mundial.

CONFLICTS OF INTEREST: The authors declare that they have no conflicts of interest.

FINANCIAL SUPPORT: This work has not received any contribution, grant or scholarship.

CONFIDENTIALITY OF DATA: The authors declare that they have followed the protocols of their work center on the publication of data from patients.

PROTECTION OF HUMAN AND ANIMAL SUBJECTS: The authors declare that the procedures followed were in accordance with the regulations of the relevant clinical research ethics committee and with those of the Code of Ethics of the World Medical Association (Declaration of Helsinki).

\section{REFERÊNCIAS}

1. Lorch MP, Whurr R. Tracing spasmodic dysphonia: the source of Ludwig Traube's priority. Ann Otol Rhinol Laryngol. 2016;125:672-6. doi: 10.1177/0003489416646115.

2. Lorch MP, Whurr R. Morell Mackenzie's contribution to the description of spasmodic dysphonia. Ann Otol Rhinol Laryngol. 2016;125:976-81. doi: 10.1177/0003489416667744.

3. Aronson AE, Brown JR, Litin EM, Pearson JS. Spastic dysphonia. II. Comparison with essential (voice) tremor and other neurologic and psychogenic dysphonias. J Speech Hear Disord. 1968;33:219-31.

4. Blitzer A, Lovelace RE, Fahn S, Brin MF, Fink ME. Electromyographic findings in focal laryngeal dystonia (spastic dysphonia). Ann Otol Rhinol Laryngol. 1985;94:591-4.

5. Jacome DE, Yanez GF. Spastic dysphonia and Meige disease. Neurology. 1980;30:349.

6. Marsden CD, Sheehy MP. Spastic dysphonia, Meige disease, and torsion dystonia. Neurology. 1982;32:1202.

7. A prevalence study of primary dystonia in eight European countries. J Neurol. 2000;247:787-92.

8. Adler $\mathrm{CH}$, Edwards BW, Bansberg SF. Female predominance in spasmodic dysphonia. J Neurol Neurosurg Psychiatry. 1997;63:688.
9. Neychev VK, Gross RE, Lehericy S, Hess EJ, Jinnah HA. The functional neuroanatomy of dystonia. Neurobiol Dis. 2011;42:185-201. doi: 10.1016/j.nbd.2011.01.026.

10. Blitzer A, Brin MF, Stewart CF. Botulinum toxin management of spasmodic dysphonia (laryngeal dystonia): a 12-year experience in more than 900 patients. Laryngoscope. 1998;108:143541.

11. Blitzer A, Brin MF, Simonyan K, Ozelius LJ, Frucht SJ. Phenomenology, genetics, and CNS network abnormalities in laryngeal dystonia: A 30-year experience. Laryngoscope. 2018;128 Suppl 1:S1-S9. doi: 10.1002/lary.27003.

12. Grillone GA, Blitzer A, Brin MF, Annino DJ, Jr. Saint-Hilaire $\mathrm{MH}$. Treatment of adductor laryngeal breathing dystonia with botulinum toxin type A. Laryngoscope. 1994;104(1 Pt 1):30-2.

13. Ludlow CL, Adler CH, Berke GS, Bielamowicz SA, Blitzer A, Bressman SB, et al. Research priorities in spasmodic dysphonia. Otolaryngol Head Neck Surg. 2008;139:495-505. doi: 10.1016/j.otohns.2008.05.624.

14. Hillel AD. The study of laryngeal muscle activity in normal human subjects and in patients with laryngeal dystonia using multiple fine-wire electromyography. Laryngoscope. 2001;111(4 Pt 2 Suppl 97):1-47. doi: 10.1097/00005537-20010400100001.

15. Blitzer A, Brin MF, Fahn S, Lange D, Lovelace RE. Botulinum toxin (BOTOX) for the treatment of "spastic dysphonia" as part of a trial of toxin injections for the treatment of other cranial dystonias. Laryngoscope. 1986;96:1300-1.

16. Watts CC, Whurr R, Nye C. Botulinum toxin injections for the treatment of spasmodic dysphonia. Cochrane Database Syst Rev. 2004:CD004327.

17. Stachler RJ, Francis DO, Schwartz SR, Damask CC, Digoy GP, Krouse HJ, et al. Clinical practice guideline: hoarseness (dysphonia). Otolaryngol Head Neck Surg. 2018;158(1_suppl):S1-S42. doi: 10.1177/0194599817751030.

18. Mor N, Simonyan K, Blitzer A. Central voice production and pathophysiology of spasmodic dysphonia. Laryngoscope. 2018;128:177-83. doi: 10.1002/lary.26655.

19. Ludlow CL. Spasmodic dysphonia: a laryngeal control disorder specific to speech. J Neurosci. 2011;31:793-7. doi: 10.1523/ JNEUROSCI.2758-10.2011.

20. Bielamowicz S, Ludlow CL. Effects of botulinum toxin on pathophysiology in spasmodic dysphonia. Ann Otol Rhinol Laryngol. 2000;109:194-203.

21. Hirano M. Psycho-acoustic evaluation of voice. In: Arnold GE WF, Wyke BD, editors. Disorders of Human Communication. Clinical Examination of Voice. Vienna: Springer-Verlag; 1981. p. 81-4.

22. Creighton FX, Hapner E, Klein A, Rosen A, Jinnah HA, Johns MM. Diagnostic delays in spasmodic dysphonia: a call for clinician education. J Voice. 2015;29:592-4. doi: 10.1016/j.jvoice.2013.10.022.

23. Berke GS, Blackwell KE, Gerratt BR, Verneil A, Jackson KS, Sercarz JA. Selective laryngeal adductor denervation-reinnervation: a new surgical treatment for adductor spasmodic dysphonia. Ann Otol Rhinol Laryngol. 1999;108:227-31.

24. Chhetri DK, Mendelsohn AH, Blumin JH, Berke GS. Long-term follow-up results of selective laryngeal adductor denervation-reinnervation surgery for adductor spasmodic dysphonia. Laryngoscope. 2006;116:635-42. doi: 10.1097/01. MLG.0000201990.97955.E4.

25. Koufman JA, Rees CJ, Halum SL, Blalock D. Treatment of adductor-type spasmodic dysphonia by surgical myectomy: a preliminary report. Ann Otol Rhinol Laryngol. 2006;115:97102. 
26. Su CY, Chuang HC, Tsai SS, Chiu JF. Transoral approach to laser thyroarytenoid myoneurectomy for treatment of adductor spasmodic dysphonia: short-term results. Ann Otol Rhinol Laryngol. 2007;116:11-8.

27. Su CY, Lai CC, Wu PY, Huang HH. Transoral laser ventricular fold resection and thyroarytenoid myoneurectomy for adductor spasmodic dysphonia: long-term outcome. Laryngoscope. 2010;120:313-8. doi: 10.1002/lary.20714.

28. Isshiki N, Tsuji DH, Yamamoto Y, lizuka Y. Midline lateralization thyroplasty for adductor spasmodic dysphonia. Ann Otol Rhinol Laryngol. 2000;109:187-93.

29. Blitzer A. Spasmodic dysphonia and botulinum toxin: experience from the largest treatment series. Eur J Neurol. 2010;17 Suppl 1:28-30. doi: 10.1111/j.1468-1331.2010.03047.x.

30. Maloney AP, Morrison MD. A comparison of the efficacy of unilateral versus bilateral botulinum toxin injections in the treatment of adductor spasmodic dysphonia. J Otolaryngol. 1994;23:160-4.

31. Bielamowicz S, Stager SV, Badillo A, Godlewski A. Unilateral versus bilateral injections of botulinum toxin in patients with adductor spasmodic dysphonia. J Voice. 2002;16:117-23.

32. Adams SG, Hunt EJ, Charles DA, Lang AE. Unilateral versus bilateral botulinum toxin injections in spasmodic dysphonia: acoustic and perceptual results. J Otolaryngol. 1993;22:1715.

33. Young N, Blitzer A. Management of supraglottic squeeze in adductor spasmodic dysphonia: a new technique. Laryngoscope. 2007;117:2082-4. doi: 10.1097/MLG.0b013e318124a97b.

34. Simpson CB, Lee CT, Hatcher JL, Michalek J. Botulinum toxin treatment of false vocal folds in adductor spasmodic dysphonia: Functional outcomes. Laryngoscope. 2016;126:118-21. doi: 10.1002/lary.25515.

35. Gama AC, Menezes LN, Maia AA, Rezende Neto AL, Oliveira JB. Voice related quality of life after botulinum toxin injection for spasmodic dysphonia. Rev Laryngol Otol Rhinol. 2010;131:39-44. 